\author{
磷酸基多孔芳香骨架材料用于提取铀离子

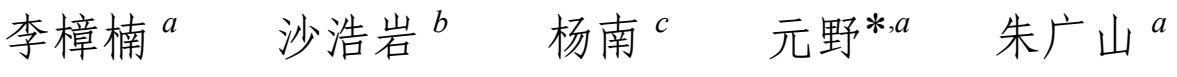 \\ $(a$ 东北师范大学化学学院 长春 130024) \\ ( ${ }^{b}$ 加州大学戴维斯分校化学工程系 美国加州戴维斯市 95616) \\ ( ${ }^{c}$ 中国一汽新能源汽车分公司 长春 130011)
}

\begin{abstract}
摘要 采用联苯为建筑基元, 合成了价格低廉、操作简便、易于工业化的多孔芳香骨架材料 PAF-45. 通过后修饰方法, 制备了带有磷酸基团的多孔芳香材料(PAF-45-PG). 通过 FTIR(傅里叶变换红外光谱), TGA(热重分析), PXRD(多晶粉末 $\mathrm{X}$ 射线衍射), SEM(扫描电子显微镜), TEM(透射电子显微镜) 以及 $\mathrm{N}_{2}$ 吸附实验, 对 PAF-45-PG 的结构及孔道性质进行了 系统的表征. 由于骨架中引入了磷酸基团, PAF-45-PG 具有优良的铀离子吸附性能, 在 $\mathrm{pH}=6$ 的条件下可以达到 100 $\mathrm{mg}^{-1}$. 另外, 该材料成本低廉, 具有可观的工业化前景, 为多孔材料在能源方面的应用提供了广阔前景.

关键词 多孔芳香骨架; 磷酸基; 肖尔反应; 铀离子提取; 工业化
\end{abstract}

\title{
Phosphoric Acid Based Porous Aromatic Framework for Uranium Extraction
}

Li, Zhangnan $^{a} \quad$ Sha, Haoyan $^{b} \quad$ Yang, Nan $^{c} \quad$ Yuan, Ye $^{* a} \quad$ Zhu, Guangshan ${ }^{a}$

( ${ }^{a}$ Key Laboratory of Polyoxometalate Science of Ministry of Education, Northeast Normal University, Changchun 130024)

( ${ }^{b}$ Department of Chemical Engineering, University of California, Davis, Davis, California 95616, United States)

( ${ }^{c}$ China Faw New Energy Vehicle Branch, Changchun 130011)

\section{1 引言}

核能源作为一种清洁、安全、高效、经济的能源，在 保证全球能源安全和应对气候变化方面具有不可替代
的作用. 国际原子能(IAEA)等机构预测：未来的几十 年, 铀核裂变产生的能量将成为全球主要的能量来源. 铀元素是核能发电的主要原料之一, 然而伴随着核电产 业的高速发展，陆地上较易开采的铀矿资源将在未来几

\footnotetext{
*E-mail: Yuany101@nenu.edu.cn
}

Received January 16, 2019; published February 14, 2019.

Supporting information for this article is available free of charge via the Internet at http://sioc-journal.cn.

Project supported by the National Basic Research Program of China (973 Program, No. 2014CB931804) and the National Natural Science Foundation of China (NSFC Project, Nos. 91622106, 21531003, 21601031).

项目受国家重点基础研究发展计划(973, No. 2014CB931804)和国家自然科学基金(Nos. 91622106, 21531003, 21601031)资助. 
十年间耗尽. 海水中溶解铀的含量高达 45 亿吨 ${ }^{[1 \sim 5]}$, 高 效大量的从海水中富集铀元素将极大程度的降低工业 中的成本和能耗, 改变现有的能源产业链, 提高工业生 产效益, 保证人类未来的能源需求. 然而, 海水中的铀 离子浓度极低约为 $3.3 \mathrm{ppb}$, 导致常规的方法很难有效 的从海水中提取铀离子. 因此, 设计和合成高效的吸附 剂材料是当前亟待解决的问题 ${ }^{[6 \sim 11]}$.

到目前为止，大量具有吸附铀功能的基团被修饰到 多孔材料的孔道中, 制备了多种吸附剂材料, 如: 甲壳 素/壳聚糖基材料 ${ }^{[12]}$ 、复合材料 ${ }^{[13,14]}$ 、金属-有机杂化

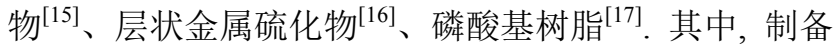
纳米零价铁复合材料, 借助协同效应增强材料的吸铀能 力, 高效环保的实现放射污染的处理 ${ }^{[18]}$; 合成含有䒺二 肟基团的纤维用于海水提铀, 吸附量可以达到 $50 \mathrm{mg}$ $\mathrm{g}^{-1[19]}$; 利用聚丙烯腈修饰多孔芳香骨架材料, 吸附量

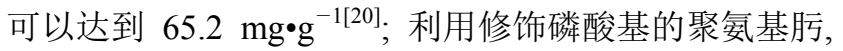
吸附量在低 $\mathrm{pH}$ 的情况下可以达到接近 $90 \mathrm{mg}^{\circ} \mathrm{g}^{-1}$ [21]. 在 众多用于吸附铀离子的基团中, 磷酸基团作为一种结合 能力强、物理化学性质稳定、易于后修饰的官能团 ${ }^{[22 ~ 28]}$, 吸引了众多化学家的广泛关注.

多孔芳香骨架材料(PAF) 是一种新型的多孔材料, 其具有比表面积大、结构刚性、孔道可调节、物理化学 性质稳定等诸多优点 ${ }^{[29]}$, 这些特性十分利于实际应用, 比如: 在 PAF 材料中负载银离子, 制备了一种高效简便 耐用的抗菌涂料 ${ }^{[30]}$; 利用分子印迹技术, 在 PAF 材料中 搭建印迹基团, 实现对铅离子的选择性去除和回收 ${ }^{[31]}$.

这里, 我们以实际的工业化为导向, 采用联苯为建 筑基元, 合成了价格低廉、可适用于工业化的多孔芳香 骨架材料 PAF-45 $5^{[32]}$ (图 1), 通过后修饰方法, 制备了带 有磷酸基团的多孔芳香材料(PAF-45-PG) ${ }^{[33]}$. 采用 FTIR, TGA, PXRD, SEM, TEM 和 $\mathrm{N}_{2}$ 吸附实验对该化合物的结 构及孔道性质进行表征. 通过铀离子的吸附实验我们观 察到, PAF-45-PG 具有优良的铀吸附性能, 在初始铀浓 度为 $7 \mathrm{ppm}, \mathrm{pH}$ 值为 6 的条件下, 吸附量可以达到 100
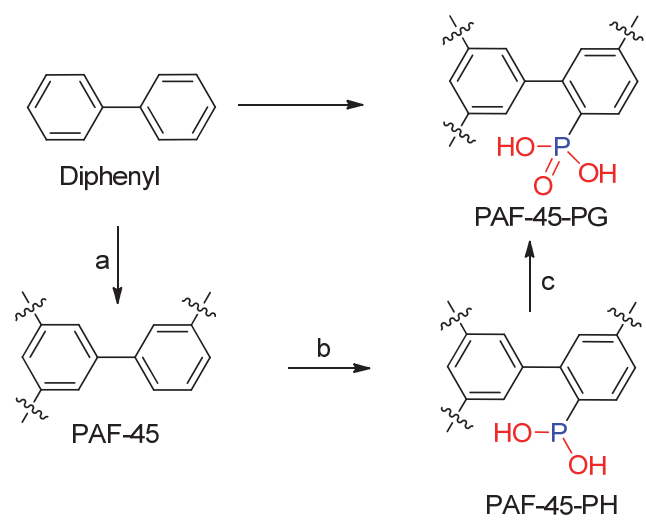

图 1 合成 PAF-45-PG 的路线图

Figure 1 Schematic illustration of synthesis of PAF-45-PG (a) $\mathrm{CHCl}_{3}, \mathrm{AlCl}_{3}$; (b) $\mathrm{NaOH} / \mathrm{HCl}$; (c) $\mathrm{H}_{2} \mathrm{O}_{2}$ $\mathrm{mg} \cdot \mathrm{g}^{-1}$. 同时该材料低廉的成本和简便的制备流程，具 有巨大的工业化前景.

\section{2 结果与讨论}

\subsection{PAF-45-PG 的红外光谱分析}

红外光谱可以很好地表征 PAF-45-PG 的键连方式, 如图 2, 与纯 PAF-45 相比, PAF-45-PG 在 $750 \mathrm{~cm}^{-1}$ 处出 现的振动峰说明了 $\mathrm{P}-\mathrm{C}$ 键的存在, 900 和 $1000 \mathrm{~cm}^{-1}$ 处 的振动峰是 $\mathrm{P}-\mathrm{OH}$ 特征双峰, $1150 \mathrm{~cm}^{-1}$ 出现的振动峰 说明了 $\mathrm{P}-\mathrm{O}$ 键的存在, 在 $1230 \mathrm{~cm}^{-1}$ 出现的振动峰表征 了磷氧双键. 总之, $700 \sim 1500 \mathrm{~cm}^{-1}$ 处出现的振动峰可 以充分地证明 PAF-45-PG 中磷酸基团的存在，这一结果 表明 PAF-45 多孔材料按照预定的路线, 通过后修饰的 方法修饰上了磷酸基团, 制得了目标产物 PAF-45-PG.

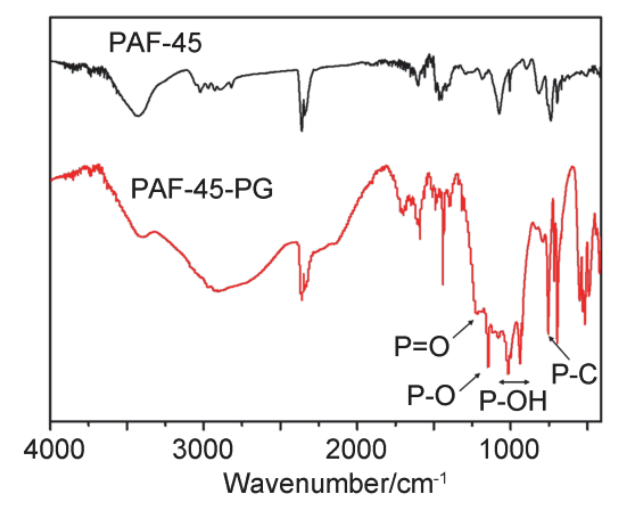

图 2 聚合物的红外图谱, PAF-45 用黑色曲线表示; PAF-45-PG 用红色 曲线表示. 扫描范围为 $400 \sim 4000 \mathrm{~cm}^{-1}$

Figure 2 FTIR spectra for polymer networks (black) of PAF-45 and (red) PAF-45-PG from $400 \sim 4000 \mathrm{~cm}^{-1}$

\subsection{X-ray 衍射和电镜分析}

粉末 $\mathrm{X}$ 射线衍射是有效的分析材料结晶性的方法. 如图 3 所示, PAF-45 和 PAF-45-PG 两种材料并没有有序 结构所特有的衍射峰. 材料的这种无定型结构可能是由 于苯环之间的相互穿插和结构扭曲导致的. 类似的无定 型的穿插和扭曲结构在有机多孔材料中经常发现. 通过 扫描电镜图片可以看出, 制备的 PAFs 材料均为球型颗 粒，其直径分布在 1 到 $3 \mu \mathrm{m}$ 之间. 如图 4 所示, 透射电 镜图片中, 我们无法观察到有序的晶格结构. 这一结论 与 $\mathrm{X}$ 射线衍射分析的结果是一致的, 即该材料为无定型 结构.

\section{3 稳定性能分析}

在空气气氛下, 我们通过热重分析仪对 PAF-45 和 PAF-45-PG 材料的热稳定性进行了测定. 如图 5 所示, 两种 PAFs 材料由室温加热到 $400{ }^{\circ} \mathrm{C}$, 这一过程中基本 无明显的重量损失. 上述实验证实了该材料具有优良的 热稳定性. 此外, 将 PAFs 材料分别浸泡在乙醇、甲醇、 


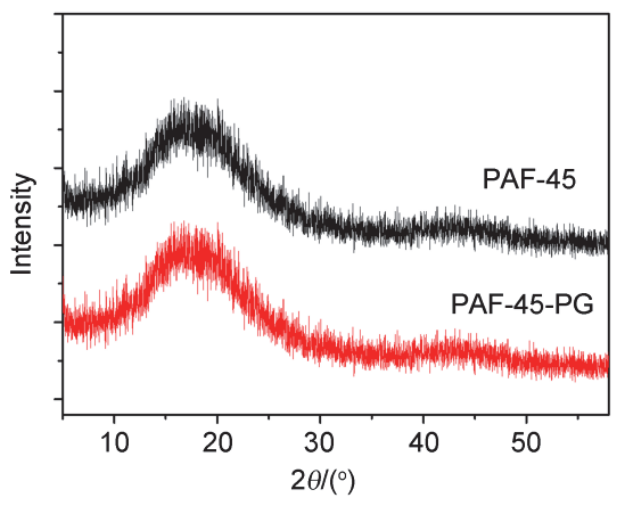

图 3 PAF-45 和 PAF-45-PG 的粉末 X 射线衍射图 Figure 3 The PXRD patterns of PAF-45 and PAF-45-PG

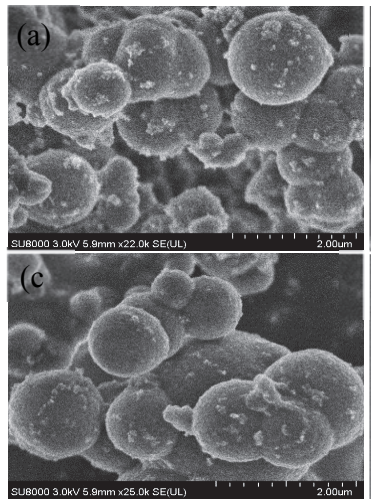

图 4 (a) PAF-45 的扫描电镜图谱; (b) PAF-45 的透射电镜图谱; (c) PAF-45-PG 的扫描电镜图谱; (d) PAF-45-PG 的透射电镜图谱

Figure 4 (a) SEM image of PAF-45; (b) HRTEM image of PAF-45; (c) SEM image of PAF-45-PG; (d) HRTEM image of PAF-45-PG

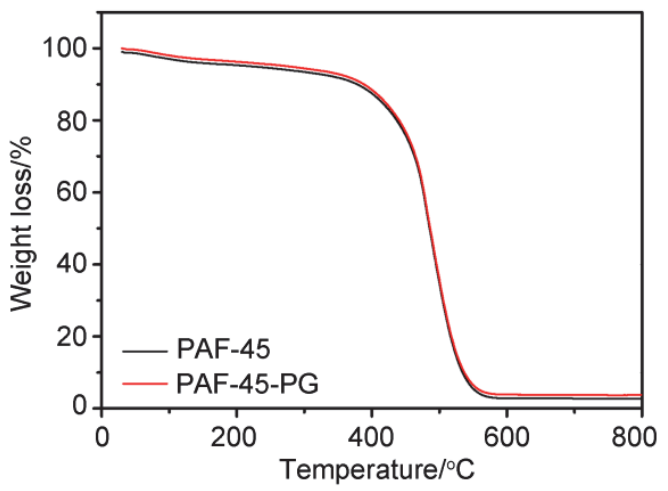

图 5 PAF-45 和 PAF-45-PG 的热重曲线

Figure 5 TGA plots for two polymer networks PAF-45 and PAF-45-PG at air condition

四氢呋喃、氯仿、丙酮和 $N, N$-二甲基甲酰胺等常规有机 溶剂中 $48 \mathrm{~h}$, 红外图谱分析证实两种材料均具有非常良 好的溶剂稳定性.

\section{4 孔道性质分析}

通过在 $77 \mathrm{~K}$ 条件下的氮气吸附, 我们对 PAFs 材料
的孔道性质进行了研究. 测试前, 我们用干燥的二氯甲 烷浸泡样品 $12 \mathrm{~h}$, 然后过滤并在 $200{ }^{\circ} \mathrm{C}$ 高真空环境下处 理 $12 \mathrm{~h}$, 将样品活化. 如图 6 所示, PAFs 均表现出 I 型的 吸附曲线特性，并且存在明显的高压区滞后. 这一现象 可以通过材料本身内部的穿插和扭曲结构进行解释. 应 用 BET 模型计算, PAF-45-PG 的比表面积为 $426 \mathrm{~m}^{2} \cdot \mathrm{g}^{-1}$. 相比于 PAF-45, 引入磷酸基团后的 PAF-45-PG 比表面 积明显下降, 这可能是由于引入了官能团, 导致空隙变 小, 进而降低了比表面积.

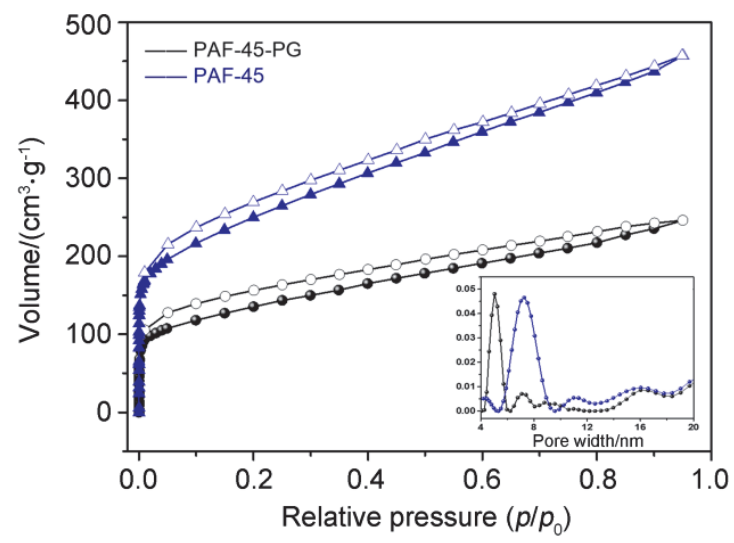

图 $6 \mathrm{~N}_{2}$ 吸附曲线(实心图标)和 $\mathrm{N}_{2}$ 脱附曲线(空心图标), 与孔径分布 Figure 6 Nitrogen gas adsorption (filled symbols)-desorption (empty symbols) isotherms, and pore size distribution

\section{5 铀吸附能力测试}

图 7 为 $20{ }^{\circ} \mathrm{C}$ 和 $\mathrm{pH}$ 为 6 条件下, PAFs 材料在模拟 海水中的吸附曲线. 经过样品在铀离子溶液中 $24 \mathrm{~h}$ 的浸 泡，我们可以看出随着铀浓度的增加 PAF-45-PG 的吸附 量也随之增大, 在 $8 \mathrm{ppm}$ 左右基本达到最大值, 为 101 $\mathrm{mg} \cdot \mathrm{g}^{-1}$. 相对应的 PAF-45 吸收峰值为 $5.9 \mathrm{mg} \cdot \mathrm{g}^{-1}$, 这一 结果表明, PAF-45-PG 对铀离子的吸附主要是其孔道中 后修饰的磷酸基官能团对铀离子的化学吸附作用, 而不 是 PAF-45-PG 本身的物理吸附作用. 图 8 为吸附动力学 曲线, 在初始铀浓度为 $7.56 \mathrm{ppm}, \mathrm{pH}$ 值为 6 的条件下进 行测试. 可以看出, 随着时间延长, PAF-45-PG 对铀的 吸附量逐渐增大，但在 $120 \mathrm{~min}$ 时基本就已达到吸附最 大值, 为 $100 \mathrm{mg} \cdot \mathrm{g}^{-1}$, 同条件下, PAF-45 对铀离子的吸 附量约为 $5 \mathrm{mg} \cdot \mathrm{g}^{-1}$, 该结果说明了 PAF-45-PG 对铀离子 的吸附作用主要来自于孔道中后修饰的磷酸基对铀离 子的化学吸附作用, 极大增强了 $\mathrm{PAF}$ 材料对铀离子的吸 附能力.

\section{6 铀吸附模型计算}

等温吸附数据与 Langmuir 和 Freundlich 模型拟合较 好, 相关系数分别大于 0.94 和 0.98 (图 9). PAF-45-PG 的 最大吸附量为 $101 \mathrm{mg} \cdot \mathrm{g}^{-1}$.

Freundlich 模型通过以下公式进行讨论: 


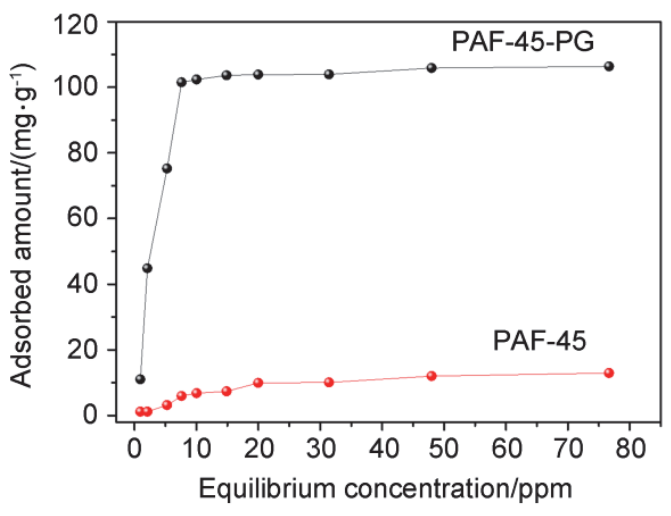

图 7 PAF-45 和 PAF-45-PG 的等温吸附曲线

Figure 7 Uranium sorption isotherms of PAF-45 and PAF-45-PG

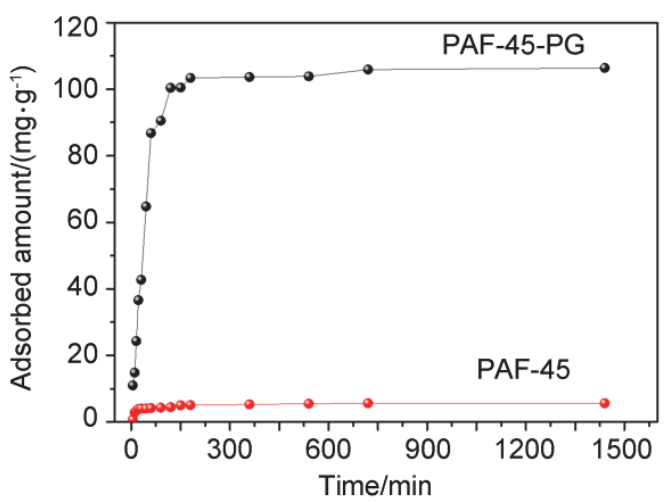

图 8 PAF-45 和 PAF-45-PG 的吸附动力学曲线

Figure 8 The kinetics of uranium adsorption of PAF-45 and PAF-45-PG

$$
\ln Q_{\mathrm{e}}=\ln K_{\mathrm{F}}+\left(\frac{1}{n}\right) \ln C_{\mathrm{e}}
$$

$Q_{\mathrm{e}}$ 为吸附量 $\left(\mathrm{mg} \cdot \mathrm{g}^{-1}\right), Q_{\mathrm{m}}$ 为最大吸附量 $\left(\mathrm{mg} \cdot \mathrm{g}^{-1}\right), K_{\mathrm{F}}$ 为 Freundlich 结合常数, $C_{\mathrm{e}}$ 为平衡浓度.

Langmuir 模型通过以下公式进行讨论:

$$
\frac{C_{\mathrm{e}}}{Q_{\mathrm{e}}}=\frac{1}{Q_{\mathrm{m}} \cdot K_{\mathrm{L}}}+\frac{C_{\mathrm{e}}}{Q_{\mathrm{m}}}
$$

$Q_{\mathrm{e}}$ 和 $C_{\mathrm{e}}$ 代表的量和 Freundlich 模型相同, $K_{\mathrm{L}}$ 为 Langmuir 结合常数. 通过拟合结果发现, PAF-45-PG 对于铀离子 的吸附作用主要来自于孔道吸附.

吸附动力学曲线符合一级反应动力学和二级反应 动力学(图 10), 拟合相关系数分别为 0.99 和 0.91 .

一级反应动力学可以通过以下公式进行计算:

$$
\ln \left(q_{\mathrm{e}}-q_{t}\right)=\ln q_{\mathrm{e}}-k_{1} t
$$

$t$ 代表吸附时间 $(\mathrm{min}), q_{\mathrm{e}}$ 代表最大吸附量 $\left(\mathrm{mg} \cdot \mathrm{g}^{-1}\right), q_{t}$ 代 表在 $t$ 时刻的吸附量 $\left(\mathrm{mg}^{\circ} \mathrm{g}^{-1}\right), k_{1}$ 为一级反应动力学吸附 常数.
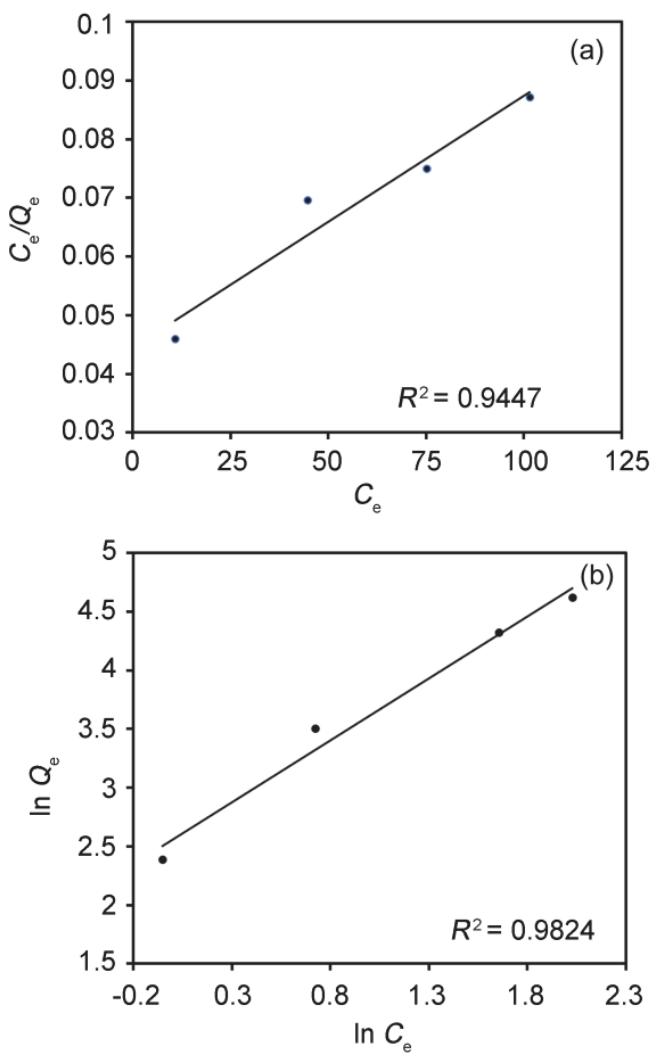

图 9 PAF-45-PG 吸附曲线的(a) Langmuir 和(b) Freandlich 模型模拟 Figure 9 Langmuir model (a) and Freandlich model (b) simulation of PAF-45-PG

二级反应动力学可以通过以下公式进行计算:

$$
\frac{t}{q_{t}}=\frac{1}{k_{2} \cdot q_{\mathrm{e}}^{2}}+\frac{t}{q_{\mathrm{e}}}
$$

$t, q_{t}$ 和 $q_{\mathrm{e}}$ 与一级反应动力学公式代表相同的量, $k_{2}$ 为二 级反应动力学常数. 经过拟合发现, 吸附动力学曲线符 合一级反应和二级反应动力学, 这说明, PAF-45-PG 对 铀离子的吸附作用主要属于化学吸附.

\section{7 洗脱和再生能力测试}

我们使用 $1 \mathrm{~mol} / \mathrm{L}$ 的碳酸钠溶液对吸附铀之后的材 料进行处理, 然后再重复进行铀吸附动力学实验, 发现 PAF-45-PG 材料很容易再生, 可以重复利用, 吸附性能 维持了至少五个循环, 其吸铀能力分别为 $99,100,98$,

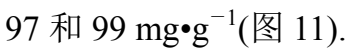

\section{3 结论}

综上所述，我们通过后修饰的方法在 PAF 材料上引 入磷酸基官能团, 成功的合成了带有磷酸基团的多孔芳 香骨架材料 PAF-45-PG. 该材料具有十分优良的物理化 学稳定性, 同时具有相对较大的比表面积. 由于成功地 引入了磷酸基团进入多孔材料中, PAF-45-PG 表现出了 

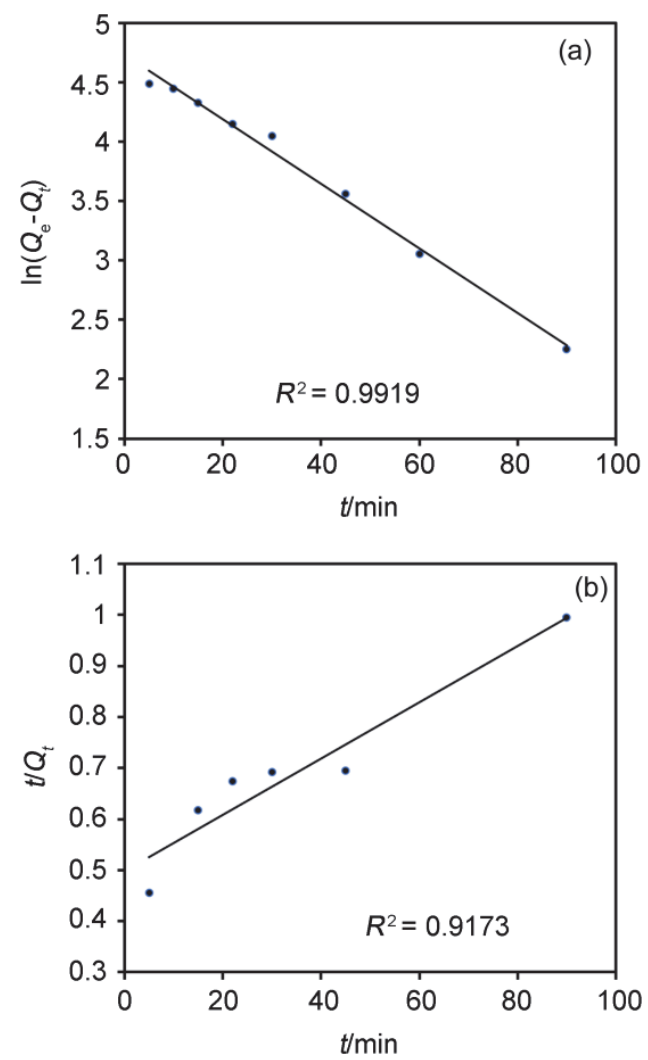

图 10 PAF-45-PG 吸附曲线的(a)一级动力学和(b)二级动力学模型模 拟

Figure 10 Pseudo-first order (a) and pseudo-second order model (b) simulation of PAF-45-PG

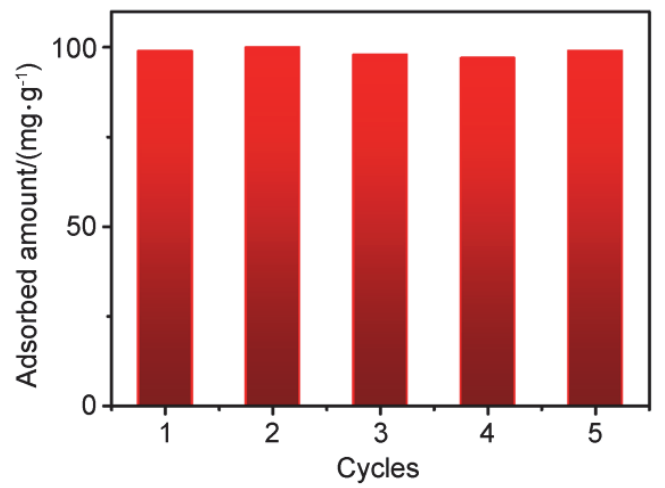

图 11 PAF-45-PG 的循环测试图

Figure 11 Cycle test patterns of PAF-45-PG

优秀的铀离子吸附容量和吸附效率. 另外, 该材料制备 成本低廉、合成路线简便, 有利于实际生产的应用, 为 多孔材料在工业应用方面提供了潜在的可能.

\section{4 实验部分}

\section{1 材料制备路线}

PAF-45 合成路线: 首先, 在 $100 \mathrm{~mL}$ 圆底烧瓶中加
入催化剂三氯化铝 $500 \mathrm{mg}$, 将烧瓶抽真空后，冲入氮 气. 接着向烧瓶中注射 $40 \mathrm{~mL}$ 氯仿, 然后 $60{ }^{\circ} \mathrm{C}$ 加热 $3 \mathrm{~h}$, 以达到活化催化剂的目的. 接着, 将 $200 \mathrm{mg}$ 联苯溶于 $20 \mathrm{~mL}$ 氯仿, 并将其注射进圆底烧瓶中. 之后, 维持 $60{ }^{\circ} \mathrm{C}$ 加热搅拌 $24 \mathrm{~h}$. 当反应体系降至常温后, 过滤得 到粗产物, 用 $1 \mathrm{~mol} / \mathrm{L}$ 盐酸, 甲醇, 丙酮, 清洗粗产物, 初步除去未反应的原料和催化剂. 然后依次用乙醇, 四 氢呋喃, 氯仿对产物进行索氏提取处理. 最终, 将得到 的产物在真空环境下, 加热到 $80{ }^{\circ} \mathrm{C}$ 持续 $24 \mathrm{~h}$, 得到 PAF-45 产品 $195.4 \mathrm{mg}$, 产率 97.7\%.

PAF-45-PG 合成路线: 在 $100 \mathrm{~mL}$ 圆底烧瓶中加入 $100 \mathrm{mg}$ 的 PAF-45 和 $1 \mathrm{~g}$ 三氯化铝, 将烧瓶抽真空后, 冲 入氮气, 然后注入 $20 \mathrm{~mL}$ 的 $2 \mathrm{~mol} / \mathrm{L}$ 三氯化磷溶液, 加 热至 $80{ }^{\circ} \mathrm{C}$ 持续 $6 \mathrm{~h}$. 待反应结束后冷却至室温, 将过滤 得到的粗产物先后用 $1 \mathrm{~mol} / \mathrm{L} \mathrm{NaOH}$ 溶液和 $1 \mathrm{~mol} / \mathrm{L} \mathrm{HCl}$ 溶液处理, 然后反复用乙醇和二氯甲烷进行多次洗涤. 接着, 将得到的产物在真空环境下, 加热至 $80{ }^{\circ} \mathrm{C}$ 持续 $24 \mathrm{~h}$, 得到 PAF-45-PH. 接着将得到的 PAF-45-PH 均匀 分散在 $30 \mathrm{~mL}$ 双氧水中，搅拌 $24 \mathrm{~h}$. 过滤得到的产物用 乙醇进行索氏提取处理, 最终, 将产物在真空环境下, 加热至 $80{ }^{\circ} \mathrm{C}$ 持续 $24 \mathrm{~h}$, 得到 PAF-45-PG.

\section{2 铀吸附能力测试}

PAF-45-PG 铀吸附能力测试:

(1)等温吸附测试: 首先, 配制浓度梯度的铀溶液各 $1 \mathrm{~L}$ (1 ppm, 2 ppm, 5 ppm, 7.5 ppm, 10 ppm, 15 ppm, 20 ppm, $30 \mathrm{ppm}, 50 \mathrm{ppm}, 75 \mathrm{ppm}$ ), 然后分别加入 $1 \mathrm{mg}$ PAF-45-PG 材料, 待吸附 $24 \mathrm{~h}$ 后, 分别取溶液, 用 0.45 $\mu \mathrm{m}$ 滤膜过滤后，得到的溶液进行 ICP 测试.

(2)吸附动力学测试: 首先, 配制 $7.56 \mathrm{ppm}$ 的铀溶液 $1 \mathrm{~L}$, 再加入 $10 \mathrm{mg}$ PAF-45-PG 材料, 在 5, 10, 15, 22, 30, $45,60,90,120,150,180,360,540,720,1440 \mathrm{~min}$, 分别 取 $5 \mathrm{~mL}$ 溶液, 用 $0.45 \mu \mathrm{m}$ 滤膜过滤后, 得到的溶液进行 ICP 测试.

PAF-45 铀吸附能力测试: 具体操作和 PAF-45-PG 相同.

支持信息(Supporting Information) 正文实验中使用 的试剂、仪器详见于“支持信息”(Supporting Information) 中.

\section{References}

[1] Kim, J.; Tsouris, C.; Mayes, R. T.; Oyola, Y.; Saito, T.; Janke, C. J.; Dai, S.; Schneider, E.; Sachde, D. Sep. Sci. Technol. 2013, 48, 367.

[2] Yue, Y.; Mayes, R. T.; Gill, G.; Kuo, L.; Wood, J.; Binder, A.; Brown, S.; Dai, S. RSC Adv. 2015, 5, 50005.

[3] Kim, J.; Tsouris, C.; Oyola, Y.; Janke, C. J.; Mayes, R. T.; Dai, S.; Gill, G.; Kuo, L.; Wood, J.; Choe, K.; Schneider, E.; Lindner, H. Ind. Eng. Chem. Res. 2014, 53, 6076.

[4] Saito, T.; Brown, S.; Chatterjee, S.; Kim, J.; Tsouris, C.; Mayes, R.T.; Kuo, L.; Gill, G.; Oyola, Y.; Janke, C. J.; Dai, S. J. Mater Chem. A 2014, 2, 14674.

[5] Kim, J.; Oyola, Y.; Tsouris, C.; Cole, C. R.; Mayes, R. T.; Janke, J. 
C.; Dai, S. Ind. Eng. Chem. Res. 2013, 52, 9433.

[6] Sholl, D. S.; Lively, R. P. Nature 2016, 532, 435.

[7] Lu, Y. Nat. Chem. 2014, 6, 175 .

[8] Yue, Y.; Mayes, R.; Kim, J.; Sun, X.; Chen, J.; Dai, S. Angew. Chem., Int. Ed. 2013, 52, 13458.

[9] Liu, C.; Xie, J.; Zhao, J.; Wu, T.; Wang, H.; Liu, W.; Zhang, J.; Cui, Y. Nat. Energy. 2017, 2, 17007.

[10] Feng, M.; Sarma, D.; Qi, X.; Du, K.; Huang, X. J. Am. Chem. Soc. 2016, 138,12578 .

[11] Sun, Q.; Aguila, B. Adv. Mater. 2018, 1705479.

[12] Barber, P. S.; Kelley, S. P.; Griggs, C. S.; Wallace, S.; Rogers, R. D. Green Chem. 2014, 16, 1828 .

[13] Yue, Y.; Sun, X.; Mayes, R. T.; Kim, J.; Fulvio, P. F.; Qiao, Z.; Brown, S.; Tsouris, C.; Oyola, Y.; Dai, S. Sci. China: Chem. 2013, $56,1510$.

[14] Kobuke, Y.; Tabushi, I.; Aoki, T.; Kamaishi, T.; Hagiwara, I. Ind. Eng. Chem. Res. 1988, 27, 1461.

[15] Carboni, M.; Abney, C. W.; Liu, S.; Lin, W. Chem. Sci. 2013, 4, 2396.

[16] Manos, M. J.; Kanatzidis, M. G. J. Am. Chem. Soc. 2012, 134, 16441.

[17] Kobayashi, S.; Tokunoh, M.; Saegusa, T.; Mashio, F. Macromolecules 1985, 18, 2357.

[18] Chen, H. J.; Huang, S. Y.; Zhang, Z. B.; Liu, Y. H.; Wang, X. K. Acta Chim. Sinica 2017, 75, 560 (in Chinese). (陈海军, 黄舒怡, 张 志宾, 刘云海, 王祥科, 化学学报, 2017, 75, 560.)

[19] Chatterjee, S.; Bryantsev, V. S.; Brown, S.; Johnson, J. C.; Grant, C.
D.; Mayes, R. T.; Hay, B. P. Ind. Eng. Chem. Res. 2016, 55, 4161.

[20] Yue, Y.; Zhang, C.; Tang, Q.; Mayes, R. T.; Liao, W.; Liao, C.; Dai, S. Ind. Eng. Chem. Res. 2016, 55, 4125.

[21] Shao, D.; Wang, X.; Ren, X.; Hu, S.; Wen, J.; Tan, Z.; Marwani, H. M. J. Ind. Eng. Chem. 2018, 67, 380.

[22] Birnbaum, J. C.; Busche, B.; Lin, Y.; Shaw, W. J.; Fryxell, G. E. Chem. Commun. 2002, 1374.

[23] Ren, X.; Yang, S.; Tan, X.; Chen, C.; Sheng, G.; Wang, X. J. Hazard. Mater. 2012, 237, 199 .

[24] Yantasee, W.; Fryxell, G. E.; Addleman, R. S.; Wiacek, R. J.; Koonsiripaiboon, V.; Pattamakomsan, K.; Xu, J.; Raymond, K. N. J. Hazard. Mater. 2009, 168, 1233.

[25] Ma, T.; Yuan, Z. Dalton Trans. 2010, 39, 9570.

[26] Venkateswarlu, S.; Yoon, M. RSC Adv. 2015, 5, 65444.

[27] Zhu, Y.; Liu, Y.; Ren, T.; Yuan, Z. Nanoscale 2014, 6, 6627.

[28] Das, S.; Pandey, A. K.; Athawale, A. A.; Natarajan, V.; Manchanda, V. K. Water Treat. 2012, 38, 1140.

[29] Yang, Y.; Yan, Z.; Wang, L.; Meng, Q.; Yuan, Y.; Zhu, G. J. Mater. Chem. A 2018, 6, 5202.

[30] Yuan, Y.; Sun, F.; Zhang, F.; Ren, H.; Jing, X.; Gao, X. Adv. Mater. 2013, 25, 6619.

[31] Yuan, Y.; Sun, F.; Li, L.; Cui, P.; Zhu, G. Nat. Commun. 2014, 4260.

[32] Li, L.-N. Ph.D. Dissertation, Jilin University, Changchun, 2015 (in Chinese). (李莉娜, 博士论文, 吉林大学, 长春, 2015.)

[33] Egawa, H.; Nonaka, T.; Ikari, M. J. Appl. Polym. Sci. 2010, 29, 2045 .

(Lu, Y.) 